\title{
Performance of Garden Pea Progenies for their Growth and Yield Characteristics in Mid Hills of Himachal Pradesh
}

\author{
Sandeep Kumar*, Viveka Katoch and Akanksha Bharti
}

Dept. of Vegetable Science and Floriculture, CSK Himachal Pradesh Krishi Vishvavidyalaya, Palampur, H.P. (176 062), India

\section{Corresponding Author}

Sandeep Kumar

e-mail: sandeepraina126@gmail.com

\author{
Article History \\ Article ID: IJEP0296 \\ Received in 29 $9^{\text {th }}$ March, 2019 \\ Received in revised form 03 ${ }^{\text {rd }}$ May, 2019 \\ Accepted in final form $21^{\text {st }}$ May, 2019
}

\begin{abstract}
Low genetic diversity acts as a major bottleneck in garden pea breeding and diverse parents are required to generate new genetic material. In the present investigation, fifteen hybrids were developed during 2014-15 by utilizing five lines (Line 12, Line 13, Line 14, Line 15 and Line 17) and three testers (Matar Ageta, Palam Triloki and Arkel) and, were evaluated along with their parents following randomized complete block design during 2015-16. Same material was evaluated in $F_{2}$ during 2016-17. Observations were recorded for pod yield and related horticultural traits on ten randomly selected plants. On the basis of mean performance, cross line 17×Palam Triloki showed significantly best results for early traits viz., first flowering and days to $50 \%$ flowering. For pod yield, best cross was line $17 \times A$ rkel in $F_{1}$. While in $F_{2}$ generation, mean performance for first picking observed best for cross line $13 \times$ Palam Triloki. For pod yield, best cross was line $17 \times$ Palam Triloki. Mean pod yield plant ${ }^{-1}$ as exhibited by cross line $17 \times$ Palam Triloki was highest. Thus, cross line $17 \times$ Palam Triloki was superior in pod yield as compared to parents involved.
\end{abstract}

Keywords: Agronomic performance, garden pea, mean value

\section{Introduction}

Garden pea (Pisum sativum L.; $2 \mathrm{n}=2 \mathrm{x}=14$ ), a member of family Leguminaceae, is one of the principal vegetable crops which is cultivated for its green pods in the temperate and subtropical areas of the world. It is the second most important food legume worldwide after Phaseolus vulgaris (Jaiswal et al., 2015). Blixt (1970) indicated Mediterranean region as its principal centre of genetic diversity while the Near East and Ethiopia as its secondary habitats.

The success of any breeding programme depends upon the genetic variability present in the germplasm (Adunga and Labuschange, 2003) which provides better chances of selecting desirable types. Therefore, the genetic reconstruction of pea germplasm is first step to identify the potential genotypes for use in breeding programme. In early group of peas, only three varieties have been recommended, thus the farmers of the state have limited choice. Few early lines have been recovered in the previous study on RIL population of a cross, NDVP-250xPalam Priya. A linextester analysis was done to determine the genetic interactions in the expression of earliness and various pod characters including pod yield. Linextester mating design was proposed by Kempthorne (1957), offer means of obtaining useful information on differential parental combinations through an assessment of overall genetic architecture of the parental lines, in relation to the characters studied.

\section{Materials and Methods}

The experiment was conducted for three consecutive years viz., 2014-15, 2015-16 and 2016-17 at Vegetable Research Farm of the Department of Vegetable Science and Floriculture, CSK HPKV, Palampur (H.P.), India. The experimental material consisted of 21 genotypes (Table 1 and 2), including five parental lines (Line 12, Line 13, Line 14, Line 15 and Line 17), three testers (Matar Ageta, Palam Triloki and Arkel) and their 15 crosses. The parents along with the hybrids were evaluated along with one standard check; Palam Triloki in randomized block design with three replications in 2014-15 with row to row and plant to plant spacing of $45 \mathrm{~cm}$ (in parents $30 \mathrm{~cm}$ ) and $10 \mathrm{~cm}$ respectively. Each experimental plot was of $3 \times 1.8 \mathrm{~m}^{2}$ size accommodating 6 rows. The promising genotypes were selected and evaluated along with three checks; Matar Ageta, Palam Triloki and Arkel in randomized block design with three replications during rabi, 2016-17. Ten plants per genotype per replication were randomly selected for recording the observations at appropriate stages of crop growth during both the seasons on characters viz., days to $50 \%$ flowering, days to first picking, pod length, pods plant, seeds pod ${ }^{-1}$, pod yield 
Table 1: List of lines (female parents) and testers (male parents)

\begin{tabular}{|c|c|c|c|}
\hline SI. No & Code. No & Name & Source \\
\hline \multicolumn{4}{|c|}{ a) Female parents } \\
\hline 1. & $\mathrm{~L}_{12}$ & Line 12 & CSKHPKV Palampur \\
\hline 2. & $\mathrm{~L}_{13}$ & Line 13 & CSKHPKV Palampur \\
\hline 3. & $\mathrm{~L}_{14}$ & Line 14 & CSKHPKV Palampur \\
\hline 4. & $\mathrm{~L}_{15}$ & Line 15 & CSKHPKV Palampur \\
\hline 5. & $\mathrm{~L}_{17}$ & Line 17 & CSKHPKV Palampur \\
\hline \multicolumn{4}{|c|}{ b) Male parents } \\
\hline 1. & $\mathrm{~T}_{1}$ & Matar Ageta & IARI, New Delhi \\
\hline 2. & $\mathrm{~T}_{2}$ & Palam Triloki & CSKHPKV, Palampur \\
\hline 3. & $\mathrm{~T}_{3}$ & Arkel & PAU, Ludhiana \\
\hline
\end{tabular}

\begin{tabular}{|c|c|c|}
\hline SI. No. & Code no. & Name of $F_{2}$ progenies \\
\hline 1. & $\mathrm{~L}_{12} \times \mathrm{T}_{1}$ & 12×Matar Ageta \\
\hline 2. & $\mathrm{~L}_{12} \times \mathrm{T}_{2}$ & 12×Palam Triloki \\
\hline 3. & $\mathrm{~L}_{12} \times \mathrm{T}_{3}$ & $12 \times$ Arkel \\
\hline 4. & $\mathrm{~L}_{13} \times \mathrm{T}_{1}$ & 13×Matar Ageta \\
\hline 5. & $\mathrm{~L}_{13} \times \mathrm{T}_{2}$ & 13×Palam Triloki \\
\hline 6. & $\mathrm{~L}_{13} \times \mathrm{T}_{3}$ & $13 \times$ Arkel \\
\hline 7. & $\mathrm{~L}_{14} \times \mathrm{T}_{1}$ & 14×Matar Ageta \\
\hline 8. & $\mathrm{~L}_{14} \times \mathrm{T}_{2}$ & 14×Palam Triloki \\
\hline 9. & $\mathrm{~L}_{14} \times \mathrm{T}_{3}$ & 14×Arkel \\
\hline 10. & $\mathrm{~L}_{15} \times \mathrm{T}_{1}$ & 15×Matar Ageta \\
\hline 11. & $\mathrm{~L}_{15} \times \mathrm{T}_{2}$ & 15×Palam Triloki \\
\hline 12. & $\mathrm{~L}_{15} \times \mathrm{T}_{3}$ & $15 \times$ Arkel \\
\hline 13. & $\mathrm{~L}_{17} \times \mathrm{T}_{1}$ & 17×Matar Ageta \\
\hline 14. & $\mathrm{~L}_{17} \times \mathrm{T}_{2}$ & 17×Palam Triloki \\
\hline 15. & $\mathrm{~L}_{17} \times \mathrm{T}_{3}$ & 17×Arkel \\
\hline
\end{tabular}

plant $^{-1}$, shelling percentage, primary branches plant ${ }^{-1}$, plant height and total soluble solids. The data were analysed for randomized block design given by Panse and Sukhatme (1984). The mean values of both the generation for each trait were subjected to statistical analysis using the model suggested by Kempthorne (1957) and Singh and Chaudhary (1977).

\section{Results and Discussion}

Analysis of variance for genotypes under study revealed significant differences among the parents for all the traits except for harvest duration in $\mathrm{F}_{1}$ and for pods per plant, pod yield per plant and shelling percentage in $F_{2}$. This indicated that parents used in present study were genetically diverse. Gupta and Singh (2004) have reported similar results.

In $F_{1}$, when comparison was done between best cross combination and the parents involved for earliness, it was observed that for days to first picking, mean value observed for cross line 17×Palam Triloki was 116.67, which was a week earlier than parents involved (Table 3) (mean value of 123.67 was exhibited by line 17 and Palam Triloki). Mean value of cross combination line $17 \times$ Palam Triloki for days to $50 \%$ flowering was 84 as compared to 89 (mean value exhibited by both line 17 and Palam Triloki), which clearly indicates earliness of the cross.

For pod yield, best cross was line $17 \times \mathrm{T}_{1}$, with mean value of 44.43 for number of pods per plant, while it was just 14.40 for line 17 and 12.67 for Arkel. Mean pod yield per plant as exhibited by cross line $17 \times \mathrm{T}_{1}$ was $192.20 \mathrm{~g}$, while it was only $69.23 \mathrm{~g}$ for line 17 and $60.60 \mathrm{~g}$ for Arkel. Thus, cross line 17x $T_{1}$ was superior in pod yield as compared to parents involved. While in $\mathrm{F}_{2}$ when comparison was done between best cross combination and the parents involved for earliness, it was observed that for days to first picking, mean value observed for cross $L_{13} \times$ Palam Triloki was 108 days, which was 1.67 days (Table 4) later than the parent, Palam Triloki (106.33) and was ten days earlier than Line 13 (119.33). Mean value of cross combination $\mathrm{L}_{13} \times$ Palam Triloki for days to $50 \%$ flowering was 69.67 days as compared to 90 and 69.33 (mean value exhibited by both line 13 and Palam Triloki respectively), which clearly indicates earliness of the cross. For pod yield, best cross was $\mathrm{L}_{17} \times$ Palam Triloki, with mean value of 26.72 for number of pods per plant, while it was just 16.52 for line 17 and 15.22 for Palam Triloki.

The availability of early pea cultivars in the market fetches better prices due to less supply and high demand. Thus, '17xPalam Triloki' was the most desirable genotype for early maturity among the other recommended genotypes. For improving garden pea, wide variations for pod length, seeds per plant, pods per plant and pod yield per plant are needed along with superior lines in hybridization programme (Katoch et al., 2016). Mean pod yield plant ${ }^{-1}$ as exhibited by cross $\mathrm{L}_{17} \times$ Palam Triloki was $134.61 \mathrm{~g}$ (Table 4), while it was only 


\begin{tabular}{|c|c|c|c|c|c|c|c|c|c|c|}
\hline Characters $\rightarrow$ & $\begin{array}{c}\text { Days } \\
\text { to } 50 \% \\
\text { flowering }\end{array}$ & $\begin{array}{l}\text { Days to first } \\
\text { picking }\end{array}$ & $\begin{array}{l}\text { Pod } \\
\text { length } \\
(\mathrm{cm}) \\
\end{array}$ & $\begin{array}{l}\text { Pods } \\
\text { plant }^{-1}\end{array}$ & $\begin{array}{l}\text { Seeds } \\
\text { pod }^{-1}\end{array}$ & $\begin{array}{l}\text { Pod yield } \\
\text { plant }^{-1}(\mathrm{~g})\end{array}$ & $\begin{array}{l}\text { Shelling } \\
\text { percent- } \\
\text { age (\%) }\end{array}$ & $\begin{array}{c}\text { Primary } \\
\text { branches } \\
\text { plant }^{-1}\end{array}$ & $\begin{array}{l}\text { Plant } \\
\text { height } \\
(\mathrm{cm})\end{array}$ & $\begin{array}{c}\text { TSS } \\
\text { ( }{ }^{\circ} \text { brix) }\end{array}$ \\
\hline $\mathrm{L}_{12}$ & 84.67 & 119.00 & 8.53 & 17.21 & 5.47 & 90.85 & 51.04 & 1.48 & 48.35 & 17.63 \\
\hline $\mathrm{L}_{13}$ & 90.00 & 119.33 & 7.76 & 19.39 & 5.24 & 101.61 & 50.17 & 1.71 & 51.36 & 15.20 \\
\hline $\mathrm{L}_{14}$ & 88.67 & 120.00 & 8.61 & 18.18 & 5.80 & 91.26 & 48.42 & 2.23 & 63.59 & 17.53 \\
\hline $\mathrm{L}_{15}$ & 86.67 & 120.00 & 8.54 & 19.80 & 5.60 & 98.15 & 50.01 & 2.02 & 55.18 & 17.80 \\
\hline $\mathrm{L}_{17}$ & 88.33 & 127.33 & 8.23 & 16.52 & 5.50 & 83.54 & 52.39 & 2.20 & 52.37 & 18.27 \\
\hline $\mathrm{T}_{1}$ & 84.33 & 119.00 & 7.98 & 13.55 & 4.90 & 67.57 & 48.54 & 1.41 & 39.89 & 17.80 \\
\hline $\mathrm{T}_{2}$ & 69.33 & 106.33 & 8.44 & 15.22 & 5.98 & 75.25 & 51.15 & 2.30 & 55.91 & 18.07 \\
\hline $\mathrm{T}_{3}$ & 91.33 & 123.33 & 8.12 & 16.30 & 6.72 & 83.75 & 50.13 & 2.13 & 62.23 & 17.33 \\
\hline Mean & 85.42 & 119.29 & 8.28 & 17.02 & 5.65 & 86.50 & 50.23 & 1.94 & 53.61 & 17.45 \\
\hline Range lowest & 69.33 & 106.33 & 7.76 & 13.55 & 4.90 & 67.57 & 48.42 & 1.41 & 39.89 & 15.20 \\
\hline Range highest & 91.33 & 127.33 & 8.61 & 19.80 & 6.72 & 101.61 & 52.39 & 2.30 & 63.59 & 18.27 \\
\hline SEm \pm & 1.54 & 2.88 & 0.18 & 1.83 & 0.29 & 9.34 & 0.99 & 0.16 & 3.38 & 0.42 \\
\hline $\mathrm{CD}(p=0.05)$ & 4.39 & 8.20 & 0.52 & 5.20 & 0.83 & 26.62 & 2.82 & 0.46 & 9.62 & 1.21 \\
\hline CV (\%) & 8.12 & 5.01 & 3.70 & 12.33 & 9.64 & 13.16 & 2.65 & 18.27 & 14.17 & 5.48 \\
\hline
\end{tabular}

\begin{tabular}{|c|c|c|c|c|c|c|c|c|c|c|}
\hline \multirow[t]{2}{*}{ Crosses } & \multicolumn{2}{|c|}{ Days to $50 \%$ flowering } & \multicolumn{2}{|c|}{ Days to first picking } & \multicolumn{2}{|c|}{ Pod length $(\mathrm{cm})$} & \multicolumn{2}{|c|}{ No. of pods plant ${ }^{-1}$} & \multicolumn{2}{|c|}{ No. of seeds plant ${ }^{-1}$} \\
\hline & $\mathrm{F}_{1}$ & $\mathrm{~F}_{2}$ & $\mathrm{~F}_{1}$ & $\mathrm{~F}_{2}$ & $\mathrm{~F}_{1}$ & $\mathrm{~F}_{2}$ & $\mathrm{~F}_{1}$ & $\mathrm{~F}_{2}$ & $\mathrm{~F}_{1}$ & $\mathrm{~F}_{2}$ \\
\hline $\mathrm{L}_{12} \times \mathrm{T}_{1}$ & 93.67 & 90.33 & 127.67 & 118.33 & 6.83 & 8.68 & 20.97 & 19.24 & 8.33 & 5.87 \\
\hline $\mathrm{L}_{12} \times \mathrm{T}_{2}$ & 88.33 & 71.67 & 121.33 & 114.00 & 8.27 & 8.62 & 24.37 & 19.10 & 8.17 & 6.00 \\
\hline $\mathrm{L}_{12} \times \mathrm{T}_{3}$ & 91.00 & 92.00 & 123.00 & 123.33 & 7.50 & 8.70 & 22.10 & 19.40 & 7.77 & 6.60 \\
\hline $\mathrm{L}_{13} \times \mathrm{T}_{1}$ & 94.67 & 86.33 & 128.33 & 119.00 & 6.90 & 8.63 & 13.63 & 15.20 & 7.13 & 6.13 \\
\hline $\mathrm{L}_{13} \times \mathrm{T}_{2}$ & 97.00 & 69.67 & 127.67 & 108.00 & 6.77 & 7.95 & 23.87 & 23.34 & 6.60 & 4.30 \\
\hline $\mathrm{L}_{13} \times \mathrm{T}_{3}$ & 93.33 & 90.08 & 127.67 & 117.33 & 6.93 & 8.09 & 26.00 & 19.67 & 6.67 & 6.23 \\
\hline $\mathrm{L}_{14} \times \mathrm{T}_{1}$ & 85.33 & 88.00 & 121.33 & 115.00 & 7.10 & 7.94 & 10.43 & 11.16 & 6.03 & 5.82 \\
\hline $\mathrm{L}_{14} \times \mathrm{T}_{2}$ & 87.00 & 80.33 & 121.33 & 111.67 & 8.30 & 7.90 & 19.60 & 13.57 & 8.00 & 5.13 \\
\hline $\mathrm{L}_{14} \times \mathrm{T}_{3}$ & 88.00 & 98.00 & 121.33 & 119.33 & 8.07 & 7.91 & 12.00 & 12.12 & 7.00 & 5.37 \\
\hline $\mathrm{L}_{15} \times \mathrm{T}_{1}$ & 96.00 & 86.00 & 127.67 & 114.00 & 6.50 & 8.04 & 32.17 & 19.53 & 5.67 & 5.23 \\
\hline $\mathrm{L}_{15} \times \mathrm{T}_{2}$ & 93.33 & 79.33 & 127.67 & 112.00 & 6.37 & 7.67 & 25.73 & 15.52 & 6.00 & 5.57 \\
\hline $\mathrm{L}_{15} \times \mathrm{T}_{3}$ & 90.00 & 92.00 & 127.00 & 123.33 & 6.57 & 7.89 & 17.47 & 14.12 & 6.10 & 5.90 \\
\hline $\mathrm{L}_{17} \times \mathrm{T}_{1}$ & 95.33 & 70.67 & 127.67 & 112.00 & 6.63 & 8.18 & 44.43 & 24.36 & 7.07 & 5.37 \\
\hline $\mathrm{L}_{17} \times \mathrm{T}_{2}$ & 84.00 & 82.33 & 116.67 & 111.00 & 6.53 & 8.45 & 30.87 & 26.72 & 6.07 & 5.77 \\
\hline $\mathrm{L}_{17} \times \mathrm{T}_{3}$ & 96.67 & 93.00 & 127.67 & 123.67 & 6.30 & 8.71 & 28.50 & 23.48 & 6.97 & 6.63 \\
\hline Range & $\begin{array}{c}84.00 \text { to } \\
97.00\end{array}$ & $\begin{array}{c}69.67 \text { to } \\
98.00\end{array}$ & $\begin{array}{c}116.67 \text { to } \\
128 . .33\end{array}$ & $\begin{array}{c}108.00 \text { to } \\
123.67\end{array}$ & $\begin{array}{c}6.30 \text { to } \\
8.30\end{array}$ & $\begin{array}{c}7.67 \text { to } \\
8.71\end{array}$ & $\begin{array}{c}10.43 \text { to } \\
44.43\end{array}$ & $\begin{array}{c}11.16 \text { to } \\
26.72\end{array}$ & $\begin{array}{l}5.67 \text { to } \\
8.33\end{array}$ & $\begin{array}{l}4.30 \text { to } \\
6.63\end{array}$ \\
\hline SEm \pm & 2.14 & 1.54 & 5.15 & 2.88 & 0.21 & 0.18 & 1.58 & 1.83 & 0.23 & 0.29 \\
\hline$C D(p=0.05)$ & 4.52 & 4.39 & 10.89 & 8.20 & 0.44 & 0.52 & 3.34 & 5.20 & 0.49 & 0.83 \\
\hline CV (\%) & 2.86 & 3.14 & 5.00 & 4.25 & 6.96 & 3.86 & 8.80 & 17.63 & 5.11 & 8.81 \\
\hline
\end{tabular}




\begin{tabular}{|c|c|c|c|c|c|c|c|c|c|c|}
\hline \multirow[t]{2}{*}{ Crosses } & \multicolumn{2}{|c|}{ Pod yield plant $^{-1}(\mathrm{~g})$} & \multicolumn{2}{|c|}{$\begin{array}{c}\text { Shelling percentage } \\
(\%)\end{array}$} & \multicolumn{2}{|c|}{$\begin{array}{c}\text { Primary } \\
\text { branches plant }{ }^{-1}\end{array}$} & \multicolumn{2}{|c|}{ Plant height $(\mathrm{cm})$} & \multicolumn{2}{|c|}{ TSS ( ${ }^{\circ}$ Brix) } \\
\hline & $F_{1}$ & $\mathrm{~F}_{2}$ & $F_{1}$ & $\mathrm{~F}_{2}$ & $F_{1}$ & $\mathrm{~F}_{2}$ & $\mathrm{~F}_{1}$ & $\mathrm{~F}_{2}$ & $F_{1}$ & $\mathrm{~F}_{2}$ \\
\hline $\mathrm{L}_{12} \times \mathrm{T}_{1}$ & 90.53 & 101.95 & 39.53 & 54.04 & 1.87 & 1.90 & 55.77 & 51.90 & 17.10 & 17.70 \\
\hline $\mathrm{L}_{12} \times \mathrm{T}_{2}$ & 110.87 & 101.17 & 57.87 & 50.52 & 1.80 & 2.10 & 63.77 & 50.69 & 17.67 & 18.93 \\
\hline $\mathrm{L}_{12} \times \mathrm{T}_{3}$ & 95.00 & 101.90 & 42.80 & 51.23 & 2.07 & 1.76 & 64.17 & 47.59 & 15.93 & 17.40 \\
\hline $\mathrm{L}_{13} \times \mathrm{T}_{1}$ & 83.53 & 75.07 & 30.87 & 52.89 & 1.60 & 1.86 & 65.57 & 47.63 & 15.90 & 18.10 \\
\hline $\mathrm{L}_{13} \times \mathrm{T}_{2}$ & 100.00 & 111.34 & 37.63 & 45.38 & 1.80 & 1.64 & 68.20 & 56.98 & 16.77 & 19.17 \\
\hline $\mathrm{L}_{13} \times \mathrm{T}_{3}$ & 104.20 & 100.77 & 38.60 & 52.66 & 2.00 & 2.13 & 69.13 & 57.91 & 16.47 & 17.80 \\
\hline $\mathrm{L}_{14} \times \mathrm{T}_{1}$ & 60.00 & 55.78 & 37.57 & 51.59 & 1.20 & 1.49 & 60.90 & 48.75 & 16.20 & 18.13 \\
\hline $\mathrm{L}_{14} \times \mathrm{T}_{2}$ & 81.13 & 69.91 & 54.90 & 50.06 & 2.00 & 1.73 & 65.30 & 53.24 & 16.13 & 17.20 \\
\hline $\mathrm{L}_{14} \times \mathrm{T}_{3}$ & 53.07 & 59.58 & 45.20 & 47.71 & 1.33 & 1.67 & 67.40 & 60.61 & 16.03 & 16.87 \\
\hline $\mathrm{L}_{15} \times \mathrm{T}_{1}$ & 147.33 & 97.23 & 34.77 & 52.26 & 2.20 & 2.00 & 69.87 & 45.15 & 15.80 & 18.40 \\
\hline $\mathrm{L}_{15} \times \mathrm{T}_{2}$ & 102.63 & 85.30 & 36.57 & 49.37 & 1.87 & 2.27 & 65.00 & 57.60 & 16.13 & 18.27 \\
\hline $\mathrm{L}_{15} \times \mathrm{T}_{3}$ & 68.60 & 76.09 & 42.63 & 51.50 & 1.60 & 1.84 & 63.67 & 47.53 & 16.07 & 17.00 \\
\hline $\mathrm{L}_{17} \times \mathrm{T}_{1}$ & 192.20 & 109.51 & 43.33 & 53.38 & 2.47 & 1.72 & 76.03 & 48.75 & 16.40 & 17.80 \\
\hline $\mathrm{L}_{17} \times \mathrm{T}_{2}$ & 140.23 & 134.61 & 41.60 & 50.35 & 2.00 & 2.43 & 66.67 & 65.13 & 17.20 & 16.53 \\
\hline $\mathrm{L}_{17} \times \mathrm{T}_{3}$ & 88.70 & 119.21 & 37.63 & 50.75 & 2.13 & 1.50 & 74.30 & 56.19 & 16.00 & 17.47 \\
\hline Range & $\begin{array}{c}53.07 \text { to } \\
192.20\end{array}$ & $\begin{array}{c}55.78 \text { to } \\
134.61\end{array}$ & $\begin{array}{c}30.87 \text { to } \\
57.87\end{array}$ & $\begin{array}{l}45.38 \text { to } \\
54.04\end{array}$ & $\begin{array}{l}1.20 \text { to } \\
2.47\end{array}$ & $\begin{array}{l}1.49 \text { to } \\
2.43\end{array}$ & $\begin{array}{l}55.77 \text { to } \\
76.03\end{array}$ & $\begin{array}{l}45.15 \text { to } \\
65.13\end{array}$ & $\begin{array}{c}15.80 \text { to } \\
17.67\end{array}$ & $\begin{array}{c}16.53 \text { to } \\
19.17\end{array}$ \\
\hline SEm \pm & 6.70 & 9.34 & 1.73 & 0.99 & 0.16 & 0.16 & 3.36 & 3.38 & 0.22 & 0.42 \\
\hline$C D(p=0.05)$ & 8.17 & 26.62 & 3.65 & 2.82 & 0.34 & 0.46 & 7.08 & 9.62 & 0.27 & 1.21 \\
\hline CV (\%) & 8.62 & 17.79 & 3.67 & 3.38 & 10.80 & 14.64 & 6.14 & 10.98 & 7.27 & 4.16 \\
\hline
\end{tabular}

$83.54 \mathrm{~g}$ for line 17 and $75.25 \mathrm{~g}$ for Palam Triloki. Thus, cross $\mathrm{L}_{17} \times$ Palam Triloki was superior in pod yield as compared to parents involved.

\section{Conclusion}

From the present study it is concluded that, for pod yield, best cross was $L_{17} \times T_{2}$, with mean value of 26.72 for number of pods plant $^{-1}$. Mean pod yield plant ${ }^{-1}$ as exhibited by cross $\mathrm{L}_{17} \times \mathrm{T}_{2}$ was $134.61 \mathrm{~g}$, whereas in $\mathrm{F}_{1}$ line $17 \times \mathrm{T}_{1}$, with mean value of 30.87 for number of pods plant ${ }^{-1}$ was the best. Thus, crosses $\mathrm{L}_{17} \times \mathrm{T}_{1}$ and $L_{17} \times T_{2}$ were superior in pod yield as compared to parents involved both in $F_{1}$ and in $F_{2}$, respectively.

\section{References}

Adunga, W., Labuschange, M.T., 2003. Association of linseed characters and its variability in different environment. Journal of Agricultural Sciences 140, 285-296.

Blixt, S., 1970. Pisum. In: Frankel, O.H., Bennet, E. (Eds.), Genetic resources in plants: their exploration and conservation. Int. Biol. Programme, Blackwell Scientific Publ. Oxford, 321-326.
Gupta, A., Singh, Y.V., 2004. Linextester analysis for early yield components in vegetable pea (Pisum sativum L.). Vegetable Science 31, 17-21.

Jaiswal, N.K., Gupta, A.K., Dewangan, H., Lavanya, G.R., 2015. Genetic variability analysis in field pea (Pisum sativum L.). International Journal of Science and Research 4, 1-2 Katoch, V., Singh, P., Devi, M.B., Sharma, A., Sharma, G.D., Sharma, J.K., 2016. Study of genetic variability, character association, path analysis and selection parameters for heterotic recombinant inbred lines of garden peas (Pisum sativum var. hortense L.) under mid-hill conditions of Himachal Pradesh, India. Legume Research 39, 163-169.

Kempthorne, O., 1957. An introduction to genetic statistics. John Wiley and Sons, New York, 468-471.

Pansey, V.G., Sukhatme, P.V., 1984. Statistical Methods for Agricultural Workers. ICAR, New Delhi, 381.

Singh, R.K., Choudhary, B.D., 1977. Linextester analysis. Biometrical methods in quantitative genetic analysis. Kalyani Publishers, New Delhi/Ludhiana, 178-185. 